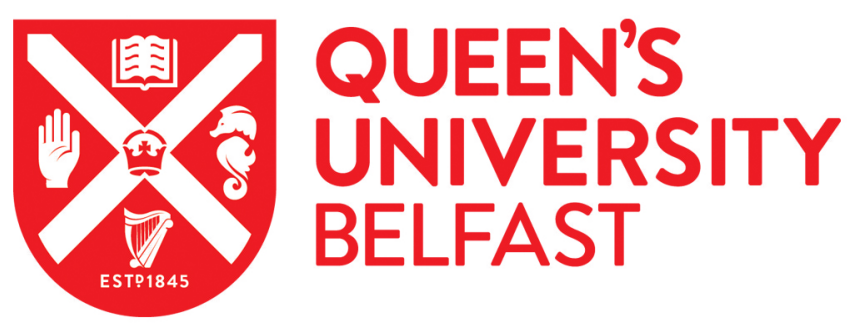

\title{
First report of the use of a saxitoxin-protein conjugate to develop a DNA aptamer to a small molecule toxin
}

Handy, S. M., Yakes, B. J., DeGrasse, J. A., Campbell, K., Elliott, C. T., Kanyuck, K. M., \& Degrasse, S. L. (2013). First report of the use of a saxitoxin-protein conjugate to develop a DNA aptamer to a small molecule toxin. Toxicon, 61(1), 30-37. https://doi.org/10.1016/j.toxicon.2012.10.015

\section{Published in:}

Toxicon

\section{Document Version:}

Peer reviewed version

Queen's University Belfast - Research Portal:

Link to publication record in Queen's University Belfast Research Portal

\section{Publisher rights}

this is the author's version of a work that was accepted for publication in Toxicon. Changes resulting from the publishing process, such as peer review, editing, corrections, structural formatting, and other quality control mechanisms may not be reflected in this document. Changes may have been made to this work since it was submitted for publication. A definitive version was subsequently published in Toxicon, VOL $61,01 / 2012$

\section{General rights}

Copyright for the publications made accessible via the Queen's University Belfast Research Portal is retained by the author(s) and / or other copyright owners and it is a condition of accessing these publications that users recognise and abide by the legal requirements associated with these rights.

Take down policy

The Research Portal is Queen's institutional repository that provides access to Queen's research output. Every effort has been made to ensure that content in the Research Portal does not infringe any person's rights, or applicable UK laws. If you discover content in the Research Portal that you believe breaches copyright or violates any law, please contact openaccess@qub.ac.uk. 


\section{Accepted Manuscript}

First report of the use of a Saxitoxin-Protein Conjugate to Develop a DNA Aptamer to a Small Molecule Toxin

Sara M. Handy, Betsy Jean Yakes, Jeffrey A. DeGrasse, Katrina Campbell, Christopher T. Elliott, Kelsey M. Kanyuck, Stacey L. DeGrasse

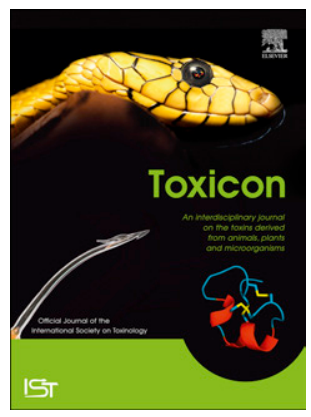

PII: S0041-0101(12)00779-9

DOI: 10.1016/j.toxicon.2012.10.015

Reference: TOXCON 4426

To appear in: Toxicon

Received Date: 31 July 2012

Revised Date: 5 September 2012

Accepted Date: 18 October 2012

Please cite this article as: Handy, S.M., Yakes, B.J., DeGrasse, J.A., Campbell, K., Elliott, C.T., Kanyuck, K.M., DeGrasse, S.L., First report of the use of a Saxitoxin-Protein Conjugate to Develop a DNA Aptamer to a Small Molecule Toxin, Toxicon (2012), doi: 10.1016/j.toxicon.2012.10.015.

This is a PDF file of an unedited manuscript that has been accepted for publication. As a service to our customers we are providing this early version of the manuscript. The manuscript will undergo copyediting, typesetting, and review of the resulting proof before it is published in its final form. Please note that during the production process errors may be discovered which could affect the content, and all legal disclaimers that apply to the journal pertain. 
First report of the use of a Saxitoxin-Protein Conjugate to Develop a DNA Aptamer to a Small Molecule Toxin

Sara M. Handy ${ }^{\mathrm{a}^{*}}$, Betsy Jean Yakes ${ }^{\mathrm{a}}$, Jeffrey A. DeGrasse ${ }^{\mathrm{a}}$, Katrina Campbell ${ }^{\mathrm{b}}$, Christopher T. Elliott ${ }^{b}$, Kelsey M. Kanyuck ${ }^{c}$, Stacey L. DeGrasse ${ }^{a}$

${ }^{a}$ U.S. Food and Drug Administration, Center for Food Safety and Applied Nutrition, 5100 Paint Branch Parkway, College Park, MD 20740, USA

${ }^{\mathrm{b}}$ Institute of Agri-Food and Land Use (IAFLU), School of Biological Sciences, Queen's University Belfast, David Keir Building, Stranmillis Road, Belfast, BT9 5AG, Northern Ireland, United Kingdom

' Joint Institute for Food Safety and Applied Nutrition (JIFSAN), University of Maryland, College Park, MD 20742, USA

*Corresponding author: sara.handy@fda.hhs.gov Tel.: 1-240-402-3063 


\section{Abstract}

Saxitoxin (STX) is a low molecular weight neurotoxin mainly produced by certain marine dinoflagellates that, along with its family of similarly related paralytic shellfish toxins, may cause the potentially fatal intoxication known as paralytic shellfish poisoning. Illness and fatality rates are low due to the effective monitoring programs that determine when toxins exceed the established regulatory action level and effectuate shellfish harvesting closures accordingly. Such monitoring programs rely on the ability to rapidly screen large volumes of samples. Many of the screening assays currently available employ antibodies or live animals. This research focused on developing an analytical recognition element that would eliminate the challenges associated with the limited availability of antibodies and the use of animals. Here we report the discovery of a DNA aptamer that targets STX. Concentration-dependent and selective binding of the aptamer to STX was determined using a surface plasmon resonance sensor. Not only does this work represent the first reported aptamer to STX, but also the first aptamer to any marine biotoxin. A novel strategy of using a toxin-protein conjugate for DNA aptamer selection was successfully implemented to overcome the challenges associated with aptamer selection to small molecules. Taking advantage of such an approach could lead to increased diversity and accessibility of aptamers to low molecular weight toxins, which could then be incorporated as analytical recognition elements in diagnostic assays for foodborne toxin detection. The selected STX aptamer sequence is provided here, making it available to any investigator for use in assay development for the detection of STX. 
Keywords: aptamer, saxitoxin, marine toxin, paralytic shellfish toxins, surface plasmon resonance 


\section{Introduction}

Saxitoxin $\left(\mathrm{C}_{10} \mathrm{H}_{17} \mathrm{~N}_{7} \mathrm{O}_{4}\right)$ is a low molecular weight toxin with a mass of $299.29 \mathrm{~g} \mathrm{~mol}^{-}$

${ }^{1}$. One of the most harmful marine toxins, saxitoxin (STX) is primarily produced by several dinoflagellates as well as certain cyanobacteria (Deeds et al., 2008). Along with dozens of related congeners, STX makes up a suite of neurotoxins, which is referred to as paralytic shellfish toxins (PSTs) and are responsible for the human syndrome paralytic shellfish poisoning (PSP; Etheridge, 2010). Molluscan bivalves represent the major vectors of PSTs to humans given their capacity to filter feed and accumulate high concentrations of the toxins; however, numerous non-traditional vectors such as crabs, lobster, and carnivorous snails have also been identified (Deeds et al., 2008). Despite seasonal toxic algal blooms in certain geographic regions, PSP cases are rare because of the effective biotoxin monitoring programs that have been implemented. In the United States, shellfish growing areas are closed to harvesting when toxins are found to be higher than the regulatory action level of $80 \mu \mathrm{g}$ STX equivalents per $100 \mathrm{~g}$ tissue.

The longstanding regulatory method for testing overall PSP toxicity is the mouse bioassay (MBA, AOAC Official Method of Analysis [OMA] 959.08); however, it has received much criticism for ethical reasons as well as a host of other issues including poor specificity, high cost, and high variability (Etheridge, 2010). Thus, recent emphasis has been placed on developing screening assays for PSTs that could reduce animal usage and provide a means of rapid, high throughput testing. Screening assays generally rely on incorporating analyte recognition elements such as native receptors (i.e., sodium channels) and produced biorecognition molecules (i.e., antibodies). Yet numerous 
challenges remain for existing analytical recognitions elements (notably antibodies), including limited availability, cost, and the continued use of animals for their production. This research sought to develop a new analytical recognition element for STX that was cost effective, did not employ the use of animals for production, and was widely available for use by the marine toxin community for assay development.

Aptamers are nucleic acids or peptides that are selected in vitro to bind to a target with high affinity and specificity due to the tertiary structure formed (Ellington and Szostak, 1990; Tuerk and Gold, 1990; Bunka and Stockley, 2006). These short ribonucleic acid (RNA) or single-stranded deoxyribonucleic acid (ssDNA) analytical recognition elements are discovered from a starting random library of $10^{12}-10^{15}$ oligonucleotide sequences via an iterative process known as systematic evolution of ligands by exponential enrichment (SELEX; Ellington and Szostak, 1990; Tuerk and Gold, 1990). Separation of target bound and unbound oligonucleotides has been performed using strategies such as affinity chromatography (Liu and Stormo, 2005), filtration (Schneider et al., 1993), SPR sensors (Misono and Kumar, 2005), and magnetic bead immobilization (Bruno and Kiel, 2002; Stoltenburg et al., 2005). Selected aptamers have been demonstrated for use in assays and platforms that have traditionally employed antibodies as analytical recognition elements. Aptamers display several important advantages over antibodies, including stability, lower cost, ease of production, and batch-to-batch consistency (Jayasena, 1999; Tombelli et al., 2005; Song et al., 2012). Further, and perhaps most important, aptamers do not depend on animal usage (live animals, cell lines, or in vivo conditions), either for development or production, making 
them an ethically favorable choice over antibodies. Another feature of aptamers is that they can be easily modified via simple chemical modifications without compromising their potential for binding to the target, thereby allowing for a wide range of applications and manipulation for use with a variety of platforms (Jayasena, 1999; Song et al., 2012).

Since their discovery, aptamers have been applied to different classes of targets: small molecules (including organic dyes, metals, drugs, carbohydrates, amino acids, nucleotides, and peptides), proteins (including enzymes, antibodies, gene regulatory factors, and lectins), as well as viruses and pathogenic bacteria (see reviews by Jayasena, 1999; Stoltenburg et al., 2007). Regardless of the class and size of the desired target, there are a number of molecular features that determine the ease with which aptamers with high specificity can be generated. The target should be present in high concentrations and with high purity. Ideal target candidates include aromatic compounds and hydrogen bond donors and acceptors (Stoltenburg et al., 2007). Furthermore, the molecule must be amenable to the method used to separate target bound/unbound oligonucleotides. In this study, a hapten-carrier complex employed for STX antibody production was used for aptamer selection. This novel strategy of using a toxin-protein conjugate for DNA aptamer selection was implemented to overcome the challenges associated with discovering aptamers to low molecular weight targets, analogous to the use of hapten-carrier complexes for enhancing immunogenicity for antibody production against small molecules that alone are generally non-immunogenic. As such, we were able to take advantage of known chemistries for coupling the hapten- 
carrier conjugate to magnetic beads for SELEX and subsequent protein digestion strategies for assessing protocol performance (Figure 1). Counter-selection was conducted to remove any ssDNA that bound to the protein carrier, leaving only those sequences specific to the STX target. To the best of our knowledge, this is the first example of a SELEX method employing a hapten-carrier conjugate to discover an aptamer.

The goal of this research was to use STX conjugated to a protein carrier (keyhole limpet hemocyanin $[\mathrm{KLH}]$ ) to develop an ssDNA aptamer that would bind to STX. Here we demonstrate (1) discovery of such an analytical recognition element using a haptencarrier conjugate, (2) concentration-dependent binding of the aptamer to surfacebound STX, (3) aptamer binding to free STX, and (4) selectivity of binding of STX to the discovered aptamer, compared to other aptamers and random ssDNA sequences. This novel approach to aptamer selection targeted for small molecule toxins offers a promising strategy that may be employed to generate a more extensive library of aptamers to marine toxins that can then be used for developing improved diagnostic assays.

\section{Experimental}

\subsection{Development of toxin-protein conjugate}

STX was conjugated to the carrier protein, keyhole limpet haemocyanin (KLH) via a modification of the Mannich reaction incorporating 2,2'-

(ethylenedioxy)bis(ethylamine), or Jeffamine, as a spacer compound in a similar manner 
to that reported previously for bovine serum albumin (Campbell et al., 2007). In brief, Jeffamine was conjugated initially to the KLH via amine coupling. KLH (10 mg) was dissolved in 2-( $N$-morpholino) ethanesulfonic acid (MES) buffer (0.5 mL, 0.05 M MES, $0.5 \mathrm{M} \mathrm{NaCl}, \mathrm{pH} 5)$. An aliquot $(250 \mathrm{~mL})$ of 1-ethyl-3-[3-

dimethylaminopropyl]carbodiimide (EDC, $20 \mathrm{mg}$ ) and $8 \mathrm{mg}$ of $\mathrm{N}$-hydroxysuccinimide (NHS) dissolved in MES buffer was added to the KLH and mixed for 5 min at room temperature. Then Jeffamine $(50 \mathrm{~mL}, 1 \mathrm{M})$ was added and the mixture allowed to react for $3 \mathrm{~h}$ at $20^{\circ} \mathrm{C}$. The Jeffamine-KLH conjugate was purified using a PD-10 column (GE Healthcare) versus PBS (pH 7.2). Jeffamine-KLH (1 mg), STX (200 mg) and 2.5\% formaldehyde $(80 \mathrm{~mL}$ ) were reacted, followed by dialysis over $24 \mathrm{~h}$ in $3 \times 4 \mathrm{~L}$ of $0.15 \mathrm{M}$ saline solution.

\subsection{STX-Jeffamine-KLH magnetic bead coupling and verification}

Following the Dynabeads Co-Immunoprecipitation Kit (Life Technologies, Grand Island, NY) instructions, $30 \mu \mathrm{g}$ of the STX-Jeffamine-KLH conjugate were covalently bound to $2 \times 10^{8}$ (3 mg) Dynabeads ${ }^{\odot}$ M-270 Epoxy. After the coupling and washing steps, the coated beads were suspended at $6.7 \times 10^{5}$ beads $/ \mu \mathrm{l}$, or $10 \mu \mathrm{g} / \mu \mathrm{l}$, in PBS-T (10 mM phosphate buffer, $2.7 \mathrm{mM} \mathrm{KCl,} 140 \mathrm{mM} \mathrm{NaCl}$, 0.05\% Tween-20, pH 7.4; Sigma, St. Louis, MO). A second batch of Dynabeads was coated with Jeffamine-KLH, as above, and was used for counter-selection.

To assess the success of the coupling procedure, forty microliters of ligandcoupled Dynabeads $(10 \mu \mathrm{g} / \mu \mathrm{l})$ were transferred to a microcentrifuge vial and washed $3 \times$ 
with $100 \mu \mathrm{l}$ of $50 \mathrm{mM}$ ammonium bicarbonate (Sigma Aldrich, St. Louis, MO). The beads were resuspended in $50 \mu \mathrm{l}$ of $9 \%$ acetonitrile in $50 \mathrm{mM}$ ammonium bicarbonate. After the addition of $1 \mu \mathrm{g}$ of the endoprotease trypsin, the reaction was allowed to proceed for 4 hours at $60^{\circ} \mathrm{C}$ before being quenched with acetic acid ( $1 \%$ final concentration). Five micrograms of both standard KLH (Sigma) and STX-Jeffamine-KLH, as well as $400 \mu \mathrm{g}$ of uncoupled Dynabeads were analyzed in the same fashion as positive and negative controls, respectively. The resultant tryptic peptides were analyzed by LC-MS/MS. Briefly, $10 \mu$ l of the peptide mixture was loaded onto a $0.15 \times 100 \mathrm{~mm}$ nanoAcquity column (Water, Milford, MA) and washed with $90 \%$ mobile phase A (0.1 M acetic acid) at a flow rate of $1 \mu \mathrm{l} / \mathrm{min}$. The bound peptides were eluted with the following gradient: $0-1 \mathrm{~min}, 20 \%$ mobile phase $B$ (0.1 M acetic acid in acetonitrile); $1-10 \mathrm{~min}, 40 \%$ mobile phase $\mathrm{B} ; 10-11 \mathrm{~min}, 70 \%$ mobile phase $\mathrm{B}$. The eluate was analyzed by a linear ion trap (LTQ) mass spectrometer (Thermo Fisher, Waltham, MA) with a data dependent acquisition of $3 \mathrm{MS} / \mathrm{MS}$ scans following a survey scan.

\subsection{Aptamer development (SELEX)}

The method used by DeGrasse (2012), as adapted from Murphy et al. (2003), was followed to develop the STX aptamer. The library consisted of a central string of 40 randomized nucleotides that were flanked by defined primer binding regions necessary for polymerase chain reaction (PCR) amplification. The library template was synthesized ( $1 \mu$ mole scale) with machine mixing for the bases within the center random sequence domain, and purified using polyacrylamide gel electrophoresis (PAGE) by Integrated 
DNA Technologies (IDT, Coralville, IA). Forward and reverse primers were also generated by IDT (Table 1), as were reverse primers with a 5' biotin label.

Ten rounds of aptamer selection were conducted starting with 5 nmoles ( $3 \mathrm{x}$ $10^{15}$ different ssDNA sequences) of the library and $50 \mu$ l of STX-Jeffamine-KLH coupled beads (3.35 $\times 10^{7}$, Table 2$)$ and following the SELEX protocol of DeGrasse (2012). This included running two PCRs per round using the same primers. Counter-selection rounds were included using Jeffamine-KLH coupled beads (no STX) for 6 of the 10 rounds. Otherwise, with the exception of performing 10 rounds of selection instead of the 14 rounds conducted by DeGrasse (2012), all other details of the protocol were the same, including reagents, instruments, PCR conditions, and sequencing of final products (see below). All secondary PCR products were visualized, at the end of each round and again all together upon completion of the entire SELEX process, on a $4 \% \mathrm{E}-\mathrm{Gel}^{\circledast}$ (Life Technologies) with an E-Gel 25bp DNA ladder (Life Technologies).

After round 10 , the PCR product was cloned using the One Shot ${ }^{\circledR}$ Top10 E. coli with the $\mathrm{TOPO}^{\circledR}$ TA cloning vector (Life Technologies) according to manufacturer instructions. The $E$. coli $(50 \mu \mathrm{l})$ was plated and grown overnight on pre-warmed $\left(37^{\circ} \mathrm{C}\right)$ LB agar plates containing $100 \mu \mathrm{g} / \mathrm{ml}$ ampicillin. The plates with a few hundred colonies were sent to GENEWIZ (South Plainfield, NJ) where 50 colonies were randomly selected for Sanger sequencing using the T3 sequencing primer that was present in the TOPO TA vector. The software program GeneiousPro (Drummond et al., 2010) was used to remove the primer and plasmid sequences from the 42 successful sequences returned 
from GENEWIZ. The trimmed 40 nt sequences were aligned in GeneiousPro and forced into zero gaps to directly compare each potential aptamer sequence nucleotide by nucleotide. Another alignment was generated in GeneiousPro using the ClustalW (Drummond et al., 2010) algorithm that allowed for gaps to find potential motifs among the sequences. The settings in GeneiousPro were: cost matrix set to IUB (a scoring matrix), a gap open penalty of 9 , and a gap extend cost of 3 .

The structures of the resulting sequences were compared using the program mfold (Zuker, 2003) with the following settings: linear DNA fold, folding temperature set to $24{ }^{\circ} \mathrm{C}$, ionic conditions for $\mathrm{Na}^{+}$and $\mathrm{Mg}^{++}$were 140 and $0 \mathrm{mM}$, respectively (Peyret, 2000), suboptimality was $5 \%$, the window parameter was set to 2 , and there was no set limit on the maximum distance between paired bases. If more than one structure was returned, the lowest $\Delta \mathrm{G}$ in $\mathrm{kcal} / \mathrm{mol}$ was selected for the comparison (SantaLucia, 1998).

\subsection{STX aptamer $\left(A P T^{S T X 1}\right)$ binding evaluation with an SPR sensor}

Standard laboratory materials for the SPR assays (e.g., sensor chips, amine coupling kit, and running buffers) were procured from Biacore (GE Healthcare, Piscataway, NJ). Nuclease free water (IDT) was used in the preparation of all reagents for SPR experiments. SPR running buffers (a. 10 mM HEPES, 150 mM NaCl, 3 mM EDTA, and $0.05 \%[\mathrm{v} / \mathrm{v}$ ] surfactant P20 buffer [HBS-EP+] and b. $10 \mathrm{mM} \mathrm{HEPES}, 150 \mathrm{mM} \mathrm{NaCl}$ [HBS-N]) were prepared from 10x concentrates.

The STX dihydrochloride used for chip formation and inhibition experiments was the FDA reference standard, currently available from the National Institute of Standards 
and Technology $(81.0 \mu \mathrm{g} / \mathrm{mL}$ free base in $20 \%$ ethanol $/ 80 \%$ water $) . \mathrm{APT}^{\mathrm{STX} 1}, \mathrm{APT}^{\mathrm{SEB} 1}$, $A P T^{\text {RAND1 }}$, and $A P T^{\text {RAND3 }}$ were synthesized by IDT (See Table 1 for sequences) for use in the SPR assay. The sequences $A P T^{\text {RAND1 }}$ and $A P T^{\text {RAND3 }}$ were produced using a random sequence generator (http://www.bioinformatics.org/sms/index.html).

\subsubsection{STX aptamer $\left(A P T^{S T X 1}\right)$ binding to surface-bound STX}

$A P T^{\text {STX1 }}$ binding to STX was evaluated with a Biacore T200 surface plasmon resonance instrument (GE Healthcare). The STX sensor chip development and instrumentation parameters used herein were detailed in Yakes et al. (2011). Briefly, a CM5 sensor chip was prepared with three active channels via EDC/NHS activation, Jeffamine coupling, ethanolamine blocking, and STX coupling as well as one reference channel via EDC/NHS activation and ethanolamine blocking. Sensor chip activity was verified with a standard STX inhibition assay (Yakes et al., 2011). The chip performed as expected in the STX inhibition assay with $8 \mu \mathrm{g} / \mathrm{mL}$ burro anti-STX yielding 80 resonance units (RU) and a decreasing response with a corresponding increase in STX concentration.

Lyophilized $\mathrm{APT}^{\mathrm{STX} 1}$ was reconstituted in HBS-EP+ to a concentration of $100 \mu \mathrm{M}$, and then diluted to $240 \mu \mathrm{g} / \mathrm{mL}$, heated at $95^{\circ} \mathrm{C}$ for $5 \mathrm{~min}$, and cooled on ice for $10 \mathrm{~min}$ prior to use. This stock was serially diluted in HBS-EP+ to yield 120, 60, 30, 15, 7.5, 3.75, $1.88,0.94$, and $0.47 \mu \mathrm{g} / \mathrm{mL}$ of aptamer. A dilution of $\mathrm{APT}^{\mathrm{STX} 1}$ was injected over the sensor chip surface at $12 \mu \mathrm{L} / \mathrm{min}$ for $90 \mathrm{sec}$ followed by a $30 \mathrm{sec}$ dissociation period. After regeneration was performed with $50 \mathrm{mM} \mathrm{NaOH}$ for $60 \mathrm{sec}$, the next analysis cycle 
was performed. Binding analysis was evaluated using the reference channel subtracted data to account for bulk refractive index variations. Within the sensorgram, a $5 \mathrm{sec}$ window, 15 sec post-sample injection stop, was used to quantify the amount of APT ${ }^{\text {STX1 }}$ binding. Studies to determine specificity of the created sensor surface for the selected aptamer versus other ssDNA (APT ${ }^{\mathrm{SEB} 1}, \mathrm{APT}^{\mathrm{RAND1}}$, and $\mathrm{APT}^{\mathrm{RAND3}}$ each assayed at $\left.10 \mu \mathrm{M}\right)$ followed the same assay procedures. In addition, a modified domoic acid (DA) sensor chip (Traynor et al., 2006) was used to evaluate APT ${ }^{\mathrm{STX} 1}$ binding to a similar, small molecule marine toxin. Accurate chip performance was first ensured by evaluating the response with a DA antibody, and the DA substrate performed as expected in a DA inhibition assay with 1:200 anti-DA yielding $400 \mathrm{RU}$ and a decreasing response with a corresponding increase in DA concentration. The STX aptamer $(240 \mu \mathrm{g} / \mathrm{mL})$ was exposed to the DA sensor chip for $90 \mathrm{sec}$ at $12 \mu \mathrm{L} / \mathrm{min}$. The curves were fit in GraphPad Prism (v. 5.02, La Jolla, CA) with a one-site, total binding model.

\subsubsection{STX aptamer (APT $\left.T^{S T X 1}\right)$ binding to free STX}

To perform studies on the aptamer binding to free STX, an antibody sensor substrate in conjugation with a direct toxin assay was performed. To create the substrate, a CM7 sensor chip was activated with EDC/NHS, polyclonal anti-STX (a generous gift from Beacon Analytical Systems [Saco, ME]; $75 \mu \mathrm{g} / \mathrm{mL}$ in pH 4.5, $10 \mathrm{mM}$ sodium acetate buffer) was coupled, and ethanolamine was used to block remaining active sites. In addition, one reference channel was incorporated via EDC/NHS activation and ethanolamine blocking. 
After determining assay conditions (HBS-N buffer, $50 \mu \mathrm{L} / \mathrm{min}$ flow rate, $180 \mathrm{sec}$ contact time, and regeneration with $10 \mathrm{mM}$ glycine $\mathrm{HCl}, \mathrm{pH} 3.0$ for $30 \mathrm{sec}$ at $30 \mu \mathrm{L} / \mathrm{min}$ ), direct toxin assays were performed. First STX was injected over the sensor surface and then STX mixed with $50 \%$ of $240 \mu \mathrm{g} / \mathrm{mL} \mathrm{APT}^{\text {STX1 }}$, prepared as for previous SPR assays HBS-N buffer, was flowed over the antibody substrate. Final concentrations of STX in solution were $10,000,5,000,2,500$, and $1,250 \mathrm{ng} / \mathrm{mL}$. Curve fits were performed in GraphPad Prism using the log(agonist) vs. response (three parameters) function.

\section{Results and Discussion}

\subsection{STX-Jeffamine-KLH magnetic bead coupling and verification}

The coupling of KLH and STX-Jeffamine-KLH to the M-270 epoxy coated Dynabeads was successful, as demonstrated by proteomic analysis. During the LCMS/MS analysis of the standard KLH, an MS/MS spectrum was observed that resulted from the fragmentation of a peptide $(811 \mathrm{~m} / \mathrm{z})$ that eluted from the column at $8.5 \mathrm{~min}$ (Figure 2A). This signature spectrum was also observed during the analysis of the Jeffamine-KLH-coupled and the STX-Jeffamine-KLH-coupled beads (Figure 2B and 2C, respectively). This spectrum was not observed in the negative control (uncoupled beads).

\subsection{Aptamer development (SELEX)}

Using the hapten-carrier conjugate as a target for SELEX also allowed us to apply an established protocol for discovering an aptamer to a protein target (DeGrasse, 2012) 
to a small molecule target. This led to the efficient discovery of $A P T^{S T X 1}$ in 10 rounds of selection. To remove candidate sequences that bound either directly to Jeffamine-KLH or to the solid support, counter-selection was aggressively employed. Before proceeding with the next round of selection, it was imperative to check the product. After each of the ten rounds of SELEX, the quality and size of the resultant enriched library (78 bases) was confirmed by running the secondary PCR product on a $4 \%$ agarose gel. The presence of a band with the proper molecular weight ( 78 bases) was the cue to proceed with the next round. Figure 3 illustrates the secondary products from all rounds. The success of the SELEX protocol was measured by the enrichment of a few sequences from a vast library of $\sim 3 \times 10^{15}$ different ssDNA sequences, in this case 31 out of 42 sequences were identical as is discussed below.

Product from the final round of aptamer selection was sequenced to reveal the selected STX-binding aptamers. Of the fifty positive bacterial colonies selected for sequencing, 42 sequences were returned (84\%). The sequences were trimmed to remove known plasmid and primer regions and were assessed for quality (i.e., proper length and sequence reaction confidence). The trimmed $40 \mathrm{nt}$ sequences were aligned both with no gaps (Figure 4A) and with adjusted parameters that allowed for gaps using ClustalW (Figure 4B). Out of the 42 sequences, 31 sequences, labeled $\mathrm{APT}^{\mathrm{STX} 1}$, were identical (73.8\%) (Table 1 and Figure 4). The remaining 11 sequences were not significantly homologous to each other with a percent identity of $2.5 \%$ (Figure 4A). In fact, the only common base was at the first position; however, in the clustal alignment 
(Figure 4B) a few common motifs were noted, but there were no more than 3 base runs and a small increase in percent identity to $7.7 \%$.

When synthesizing $A P T^{S T X 1}$ to evaluate binding, the priming sites were included, not only to enable PCR amplification, but also because the primers may play a role in structural stability or conformation. The sequence of $A P T^{S T X 1}$ was reported as 5'-GGT ATT GAG GGT CGC ATC CCG TGG AAA CAT GTT CAT TGG GCG CAC TCC GCT TTC TGT AGA TGG CTC TAA CTC TCC TCT (40 nt consensus in bold). The lowest free energy secondary structure of $\mathrm{APT}^{\mathrm{STX} 1}$ was predicted based on conditions during aptamer development (Figure 5). The estimated secondary structure for $\mathrm{APT}^{\mathrm{STX1}}$ revealed four stem and loop segments, two involving the primers. The secondary structures of the other 11 sequences were also predicted. Again, the sequences were not significantly homologous to each other or APT ${ }^{\mathrm{STX} 1}$, but many (8) of the structures as predicted by mfold did include a stem and loop structure that could possibly play a role in aptamer function (data not shown).

\subsection{Binding evaluation of $A P T^{S T X 1}$ using SPR sensors}

To demonstrate binding, varying concentrations of $\mathrm{APT}^{\mathrm{STX1}}$ were exposed to a well-established STX sensor chip (Yakes et al., 2011; Yakes et al., 2012). While this chip has been optimized for PST antibody evaluation, binding of APT ${ }^{\text {STX1 }}$ to the STX substrate was seen. APT ${ }^{S T X 1}$ bound to the STX surface, with the change in RU directly correlating with the amount of $\mathrm{APT}^{\mathrm{STX} 1}$ in solution (Figure 6). This increase in signal with an increase in solution $\mathrm{APT}^{\mathrm{STX} 1}$ concentration was reproducible, with low error and no dependence 
on sample order or injection. As a negative control, the APT ${ }^{\mathrm{STX} 1}$ was exposed to the reference channel, and no change in response was observed (data not shown); as such, non-specific binding of APT ${ }^{S T X 1}$ to the chip was ruled out.

To determine whether other ssDNA molecules would non-specifically bind to the sensor chip surface, several ssDNA sequences of the same length were exposed to the STX chip. APT ${ }^{\mathrm{SEB} 1}, \mathrm{APT}^{\mathrm{RAND1}}$, and $A P T^{\text {RAND3 }}$ did not exhibit binding to the STX sensor surface (data not shown), indicating that the STX sensor surface selectively bound to $A P T^{S T X 1}$. A modified DA sensor chip was used to evaluate specificity of APT ${ }^{S T X 1}$ binding relative to another small molecule marine toxin that often co-occurs in regions where STX may be present. After accurate chip performance was verified using an anti-DA/DA inhibition assay, $\mathrm{APT}^{\mathrm{STX1}}$ was exposed to the DA sensor surface. Binding to the substrate was not observed (i.e., approx. 1 RU Fc2-1 response), thus indicating that $\mathrm{APT}^{\mathrm{STX} 1}$ did not cross-react with DA (data not shown) and further supporting that the selected aptamer was specific to STX.

Finally, to show the selected aptamer has the ability to bind to free STX, an assay that detects STX from solution was developed. The highly-coupled antibody substrate (approx. 40,000 RU of anti-STX) has the ability to bind STX from solution and has an operating range of approx. 1,000 to $25,000 \mathrm{ng} \mathrm{STX} / \mathrm{mL}$ with responses between 10 and $50 \mathrm{RU}$. When the STX solutions were pre-mixed with APT ${ }^{\mathrm{STX} 1}$ and then injected over the antibody surface, no binding of STX to the sensor surface was observed (Figure 7). The lack of response in the presence of the aptamer indicated that the STX in solution was 
bound to the aptamer and was not available to bind to the antibody surface. Together, these data demonstrated that the selected aptamer not only binds to surface-bound STX, but also to free STX in solution. Such assay versatility, along with potential selectivity, suggests that aptamers may be able to outcompete antibodies in small marine toxin diagnostics. Efforts to design detection systems with the STX aptamer as well as expand our aptamer library are underway.

\section{Conclusions}

To our knowledge, we have discovered the first aptamer to a marine toxin and demonstrated that it binds selectively to STX in a concentration-dependent manner. For discovery of $\mathrm{APT}^{\mathrm{STX} 1}$, we employed a novel approach of using a hapten-carrier conjugate as the SELEX target. Coupling a small molecule to a solid support often poses a challenge to aptamer development. In particular, it can be difficult to assess the efficiency of such a coupling reaction. To avoid such a challenge, we took advantage of a hapten-carrier (STX-Jeffamine-KLH) conjugate that was produced originally for antibody production. The conjugate essentially converted a small molecule ( $299 \mathrm{Da})$ into a protein moiety ( 350 kDa), thus allowing conventional surface chemistries (e.g., amine/epoxy reaction) to be used for coupling the conjugate to a solid support. Additionally, we were able to verify the coupling reaction to ensure that the paramagnetic beads were coated with the conjugate. This assessment was crucial given that the hapten-carrier reaction reduces the number of free amines on the carrier available for coupling to the epoxy active sites on the Dynabeads. 
This study represented the first step in aptamer development for marine toxins and resulted in a promising strategy for continued aptamer selections to target marine toxins. These aptamers can be developed in a very short period of time (weeks compared to the months required for antibody production), are inexpensive to produce, and have the potential for implementation into a wide range of detection assays and platforms. While antibodies are often proprietary, this work reports the sequence of the aptamer selected to STX, thereby making it available to any researcher who wishes to employ it as an analytical recognition element in their own assay development. Future research will include discovering aptamers to other relevant PSTs and incorporating these analytical recognition elements into operational assays. It is anticipated that the use of aptamers in marine toxin detection methods could reduce or eliminate animalbased models, as well as the numerous challenges associated with antibody-based methods.

\section{Acknowledgements}

The authors would like to thank Sarah Stadig for technical assistance and Dr. John Callahan for guidance and review of the manuscript and funding for the work in Belfast from the Department of Employment and Learning via the All-island Research Initiative (ASSET Project). Thank you also to Titan Fan from Beacon Analytical for providing purified anti-STX for SPR experiments. 


\section{Ethical statement}

The authors declare that the study reported in the manuscript has been conducted in accordance with Elsevier ethical guidelines.

\section{Conflict of interest statement}

The authors declare no competing interests. 


\section{References}

Bunka, D.H.J., Stockley, G., 2006. Aptamers come of Age- At Last. Nat. Rev. Microbiol., 4, 588-596.

Bruno, J.G., Kiel, J.L., 2002. Use of magnetic beads in selection and detection of biotoxin aptamers by electrochemiluminescence and enzymatic methods. Biotechniques $32,178-+$

Campbell, K., Stewart, L. D., Doucette, G. J., Fodey, T. L., Haughey, S. A., Vilario, N., Kawatsu, K., Elliott, C. T., 2007. Assessment of specific binding proteins suitable for the detection of paralytic shellfish poisons using optical biosensor technology. Anal. Chem. 79(15), 5906-5914.

Deeds, J. R., Landsberg, J. H., Etheridge, S. M., Pitcher, G. C., Longan, S. W., 2008. Nontraditional vectors for paralytic shellfish poisoning. Marine Drugs 6, 308-348.

DeGrasse, J. A., 2012. A single-stranded DNA aptamer that selectively binds to Stapholococcus aureus enterotoxin B. PLoS ONE 7(3): e33410. doi:10.1371/ journal.pone.0033410

Drummond, A. J., Ashton, B., Buxton, S., Cheung, M., Cooper, A., Heled, J., Kearse, M., Moir, R., Stones-Havas, S., Sturrock, S., Thierer, T., Wilson, A., 2010. Geneious v5.1, available from http://www.geneious.com.

Ellington, A. D., Szostak, J. W., 1990. In vitro selection of RNA molecules that bind specific ligands. Nature 346, 818-822.

Etheridge, S. M., 2010. Paralytic shellfish poisoning: Seafood safety and human health perspectives. Toxicon 56, 108-122. 
Jayasena, S. D., 1999. Aptamers: An emerging class of molecules that rival antibodies in diagnostics. Clinical Chemistry 45(9), 1628-1650.

Liu, J., Stormo, G. D., 2005. Combining SELEX with quantitative assays to rapidly obtain accurate models of protein-DNA interactions. Nucleic Acids Research 33(17), e141

Misono, T. S., Kumar, P. K. R., 2005. Selection of RNA aptamers against human influenza virus hemagglutinin using surface plasmon resonance. Anal. Chem. 342, 312-317.

Murphy, M. B., Fuller. S. T., Richardson, P. M., Doyle, S. A., 2003. An improved method for the in vitro evolution of aptamers and applications in protein detection and purification. Nucleic Acids Research 31: e110.

Peyret, N., 2000. Prediction of Nucleic Acid Hybridization: Parameters and Algorithms PhD dissertation, Wayne State University, Department of Chemistry, Detroit, MI SantaLucia, J., 1998. A unified view of polymer, dumbbell, and oligonucleotide DNA nearest-neighbor thermodynamics. Proc. Natl. Acad Sci. USA 95, 1460-1465.

Schneider, D., Gold, L., Platt, T., 1993. Selective enrichment of RNA species for tight binding to Escherichia coli rho factor. FASEB Journal 7(1), 201-207.

Song, K. M., Lee, S., Ban, C., 2012. Aptamers and their biological applications. Sensors $12,612-631$.

Stoltenburg, R., Reinemann, C., Strehlitz, B., 2005. FluMag-SELEX as an advantageous method for DNA aptamer selection. Anal. Bioanal. Chem. 383, 83-91. 
Stoltenburg, R. Reinemann, C., Strehlitz, B., 2007. SELEX - A (r)evolutionary method to generate high-affinity nucleic acid ligands. Biomolecular Engineering 24, 381403.

Tombelli, S., Minunni, A., Mascini, A., 2005. Analytical applications of aptamers. Biosensors \& Bioelectronics 20, 2424-2434.

Tuerk, C., Gold, L., 1990. Systematic evolution of ligands by exponential enrichment: RNA ligands to bacteriophage T4 DNA polymerase. Science $249,505-510$.

Traynor, I.M., Plumpton, L., Fodey, T.L., Higgins, C., Elliott, C.T., 2006. Immunobiosensor detection of domoic acid as a screening test in bivalve molluscs: comparison with liquid chromatography-based analysis. J AOAC Int. 3, 868-72.

Yakes, B. J., Prezioso, S., Haughey, S. A., Campbell, K., Elliott, C. T., DeGrasse, S. L., 2011. An improved immunoassay for detection of saxitoxin by surface plasmon resonance biosensors. Sensors and Actuators B-Chemical 156, 805-811.

Yakes, B. J., Prezioso, S, DeGrasse, S. L. in press. Developing improved immunoassays for paralytic shellfish toxins: The need for multiple, superior antibodies. Talanta. http://dx.doi.org/10.1016/j.talanta.2012.06.073

Zuker, M., 2003. Mfold web server for nucleic acid folding and hybridization prediction. Nucl Acid Res. 31, 3406-3415. 
Figure captions:

Fig. 1: Schematic of aptamer development. The iterative process began with the STXJeffamine-KLH coupled magnetic beads being incubated with the random ssDNA library. Following incubation, bound versus unbound ssDNA were separated and then the bound SSDNA were eluted from the STX-Jeffamine-KLH coupled magnetic beads, replicated by PCR, and used as an enriched library for the next round of selection. During rounds 4-10 an additional step was included for counter-selection. Magnetic beads coupled to Jeffamine-KLH were incubated with the PCR product from the previous round. Only the SsDNA that did not bind to the beads coupled with Jeffamine-KLH were used for the next round of selection. This process consisted of a total of 10 rounds

Fig. 2: LC-MS/MS confirmation of ligand coupling to Dynal M-270 expocy-coated beads. The MS/MS spectrum of parent ion $811 \mathrm{~m} / \mathrm{z}$ was observed in $(A)$ standard $\mathrm{KLH},(\mathrm{B}) \mathrm{KLH}$-coupled beads, and (C) STX-Jeffamine-KLH coupled beads. This spectrum was not observed with the negative control of uncoupled Dynabeads.

Fig. 3: Gel image of all 10 rounds of aptamer selection showing the products from the $2^{\text {nd }} P C R$. Lane 1 is the ladder (25bp-500bp), lane $2-11$ are rounds $1-10$ respectively, and lane 12 is a no template control. 
Fig. 4: Alignments generated in GeneiousPro representing the 42 clonal sequences returned (with the primer and plasmid regions trimmed). Sequence 1 is APT ${ }^{\text {STX1 }}$ and represents 31 clones with $100 \%$ matches to each other. All other recovered sequences are shown (with no identical matches). Common bases are marked with a darker square. Panel A represents a manually edited alignment where each base was forced into position with no gaps. Panel B is a ClustalW alignment allowed for gaps to explore potential motifs.

Fig. 5: Predicted folding and structure of $\mathrm{APT}^{\mathrm{STX1}}$, as generated by the mfold web server (Zuker, 2003). Bases that were similar in all 12 sequences are highlighted in gray.

Fig. 6: SPR calibration curves showing the concentration-dependent binding of the STX aptamer $\left(A P T^{S T X 1}\right)$. (A) Raw response (in RU) from flow cell 2 with reference subtraction (flow cell 1 ) for a concentration series of $A P T^{\mathrm{STX} 1}$ and $(\mathrm{B})$ average normalized response from four replicate measurements. Curves were fit in GraphPad Prism with a one-site, total binding model.

Fig. 7: SPR calibration curves showing: (1) STX direct binding to an STX antibody sensor and (2) inhibition of STX binding when APT ${ }^{\mathrm{STX} 1}$ was added to the analyte solution prior to injection over the antibody substrate. Each data point is the 
average response from two replicate measurements (standard deviation shown by the error bars) of blank subtracted data, normalized to STX 10,000 ng/mL. 
Highlights:

- We present the first reported aptamer selected to a marine biotoxin (saxitoxin).

- A novel strategy of using a toxin-protein conjugate was utilized.

- Initial step towards new aptamers and their application to marine toxin assays. 
Table 1: The primer and library sequences used in this study as well as the sequence of the STX aptamer $\left(\mathrm{APT}^{\mathrm{STX} 1}\right)$ discovered in this work. APT ${ }^{\mathrm{STX} 1}$ was reported in 31 out of 42 sequences. Also two random sequences $\left(A P T^{\text {RAND1 }}\right.$ and $A P T^{\text {RAND3 }}$ ) were generated and used for experiments to evaluate selectivity of discovered sequences to the target.

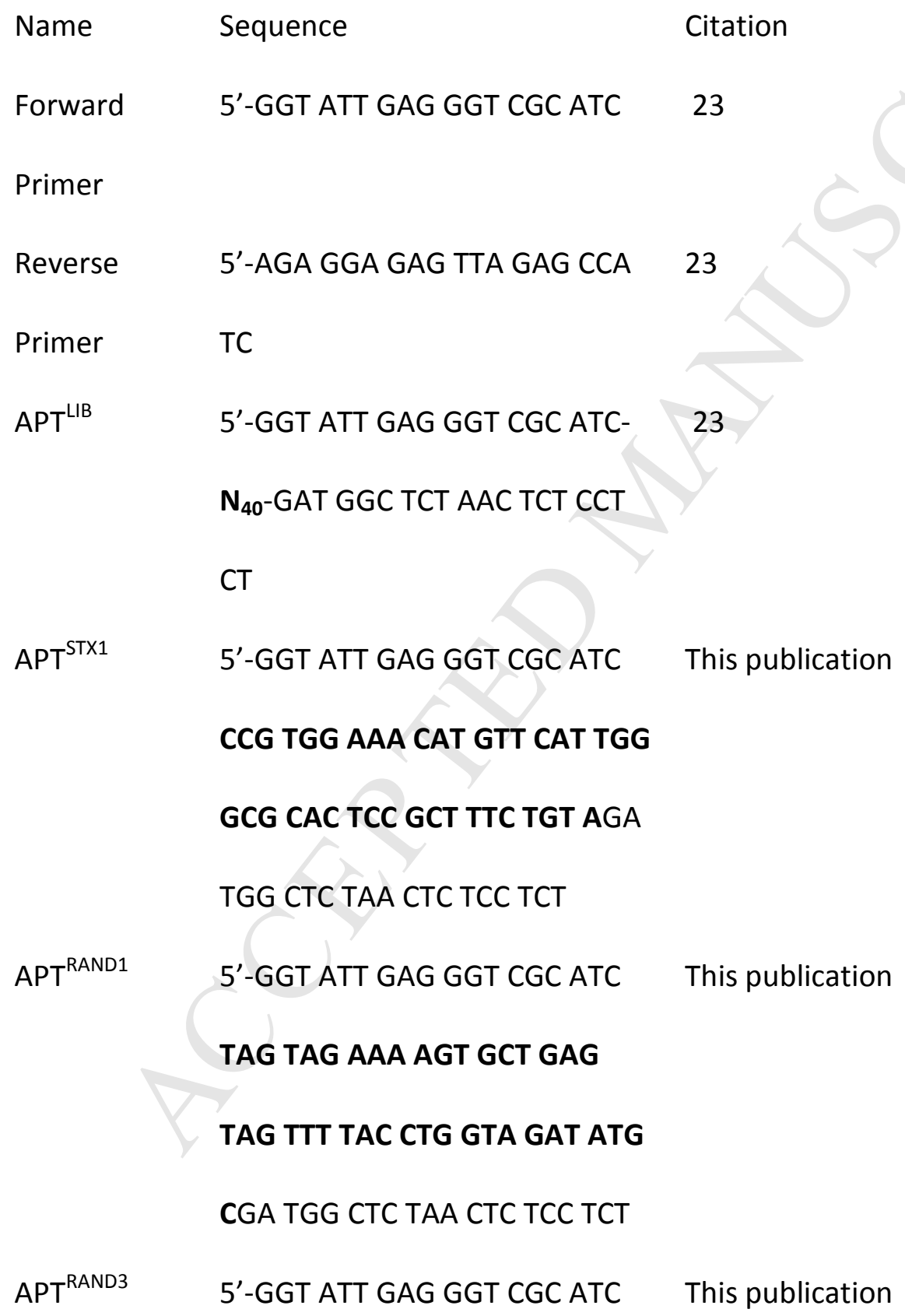


CCT CAC TTA ACA GCC CGG TCA

TGC TGA GTG TTA TTA GCT CGA

TGG CTC TAA CTC TCC TCT

$A P T^{\text {SEB1 }}$

5'-GGT ATT GAG GGT CGC ATC

23

CAC TGG TCG TTG TTG TCT GTT

GTC TGT TAT GTT GTT TCG TGA

TGG CTC TAA CTC TCC TCT 
Table 2: Values for the number of beads and incubation times used in each round. The values were modulated in later rounds to increase the stringency of the SELEX protocol.

Round Counter- Selection Incubation

Selection Beads Time (min)

1 Beads

$2-3$

0

$3.35 \times 10^{7}$

30

4-6

$3.35 \times 10^{7}$

$6.7 \times 10^{6}$

10

7-9

$3.35 \times 10^{7}$

$2.01 \times 10^{6}$

10

10

$3.35 \times 10^{7}$

$6.7 \times 10^{5}$

10 

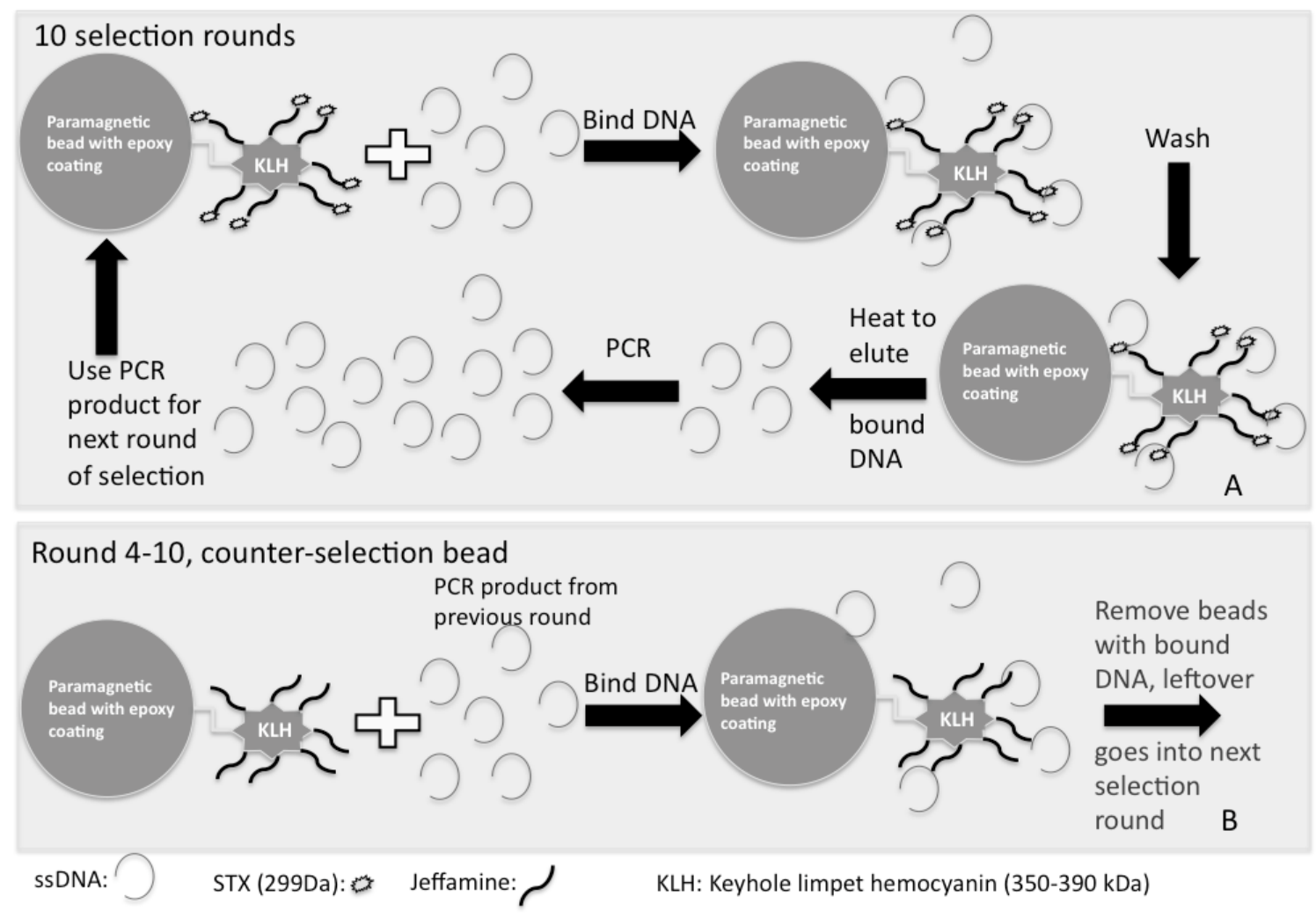
\begin{tabular}{l|l} 
RT: $8.5 \mathrm{~min}$ & Standard KLH
\end{tabular}

Parent lon: $811 \mathrm{~m} / \mathrm{z}$
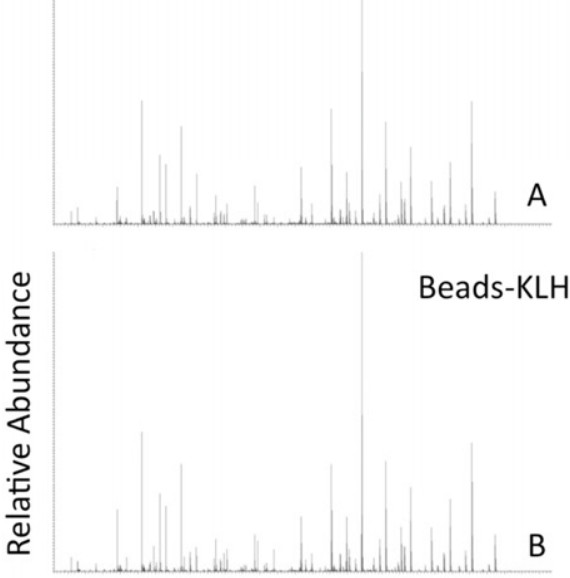

Beads-KLH-STX

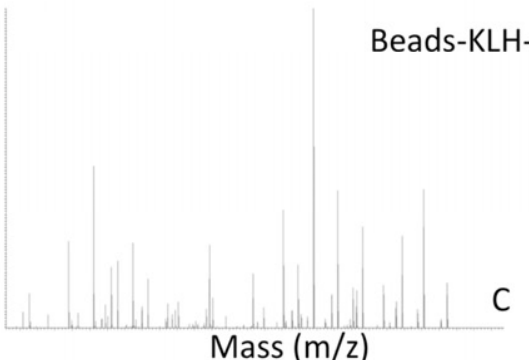




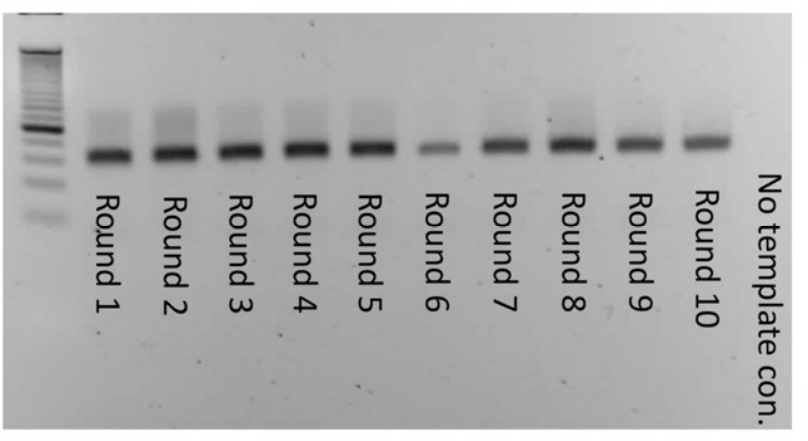




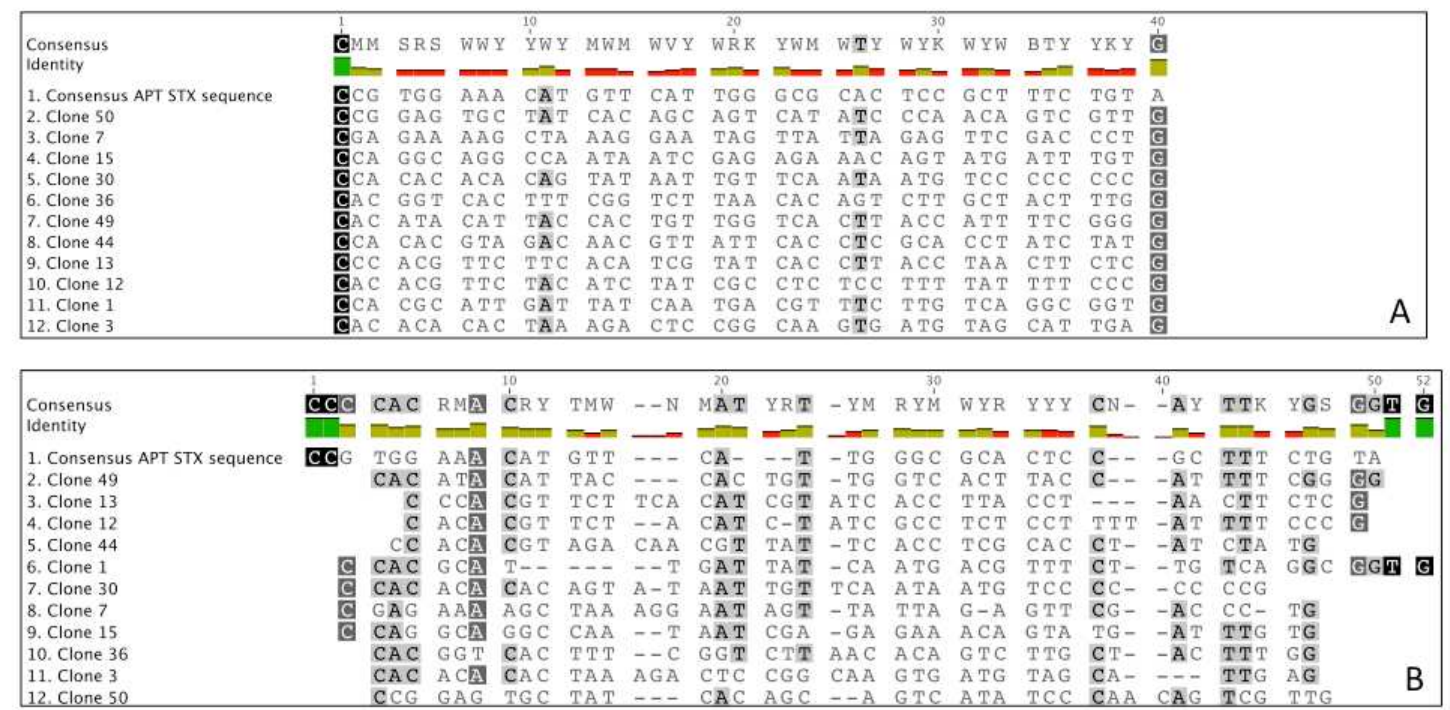




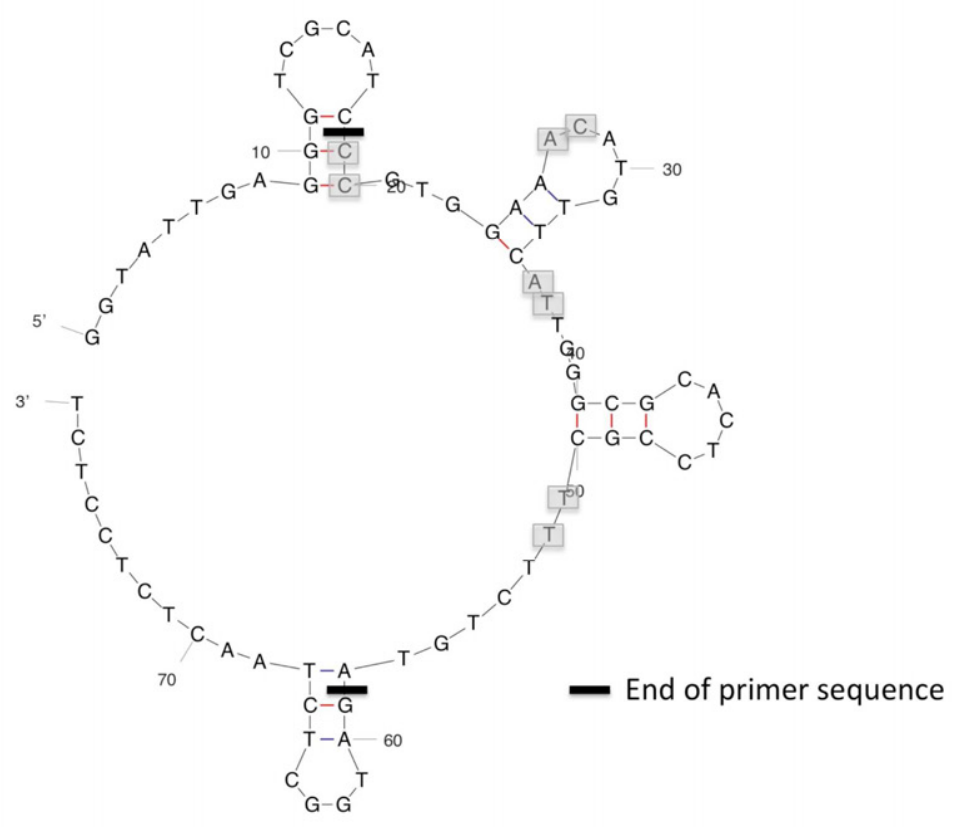


ACCEPTED MANUSCRIPT

A

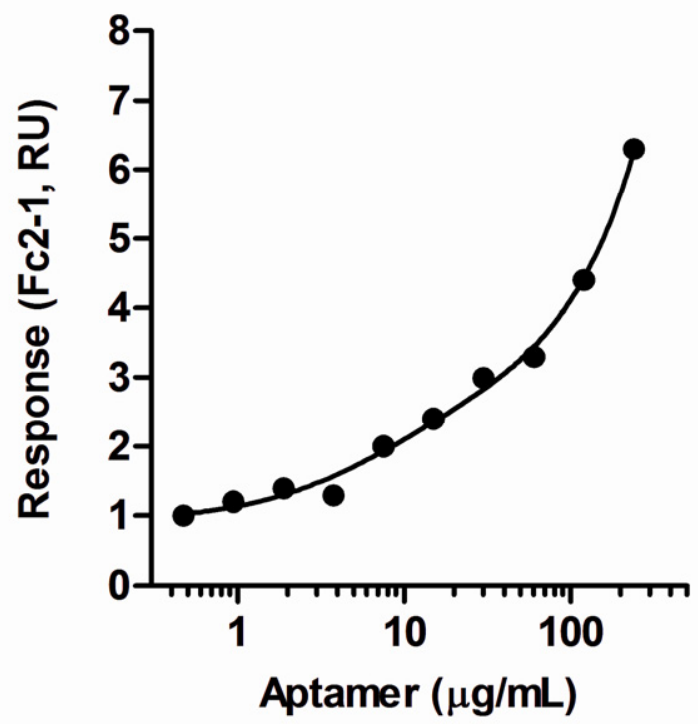

B

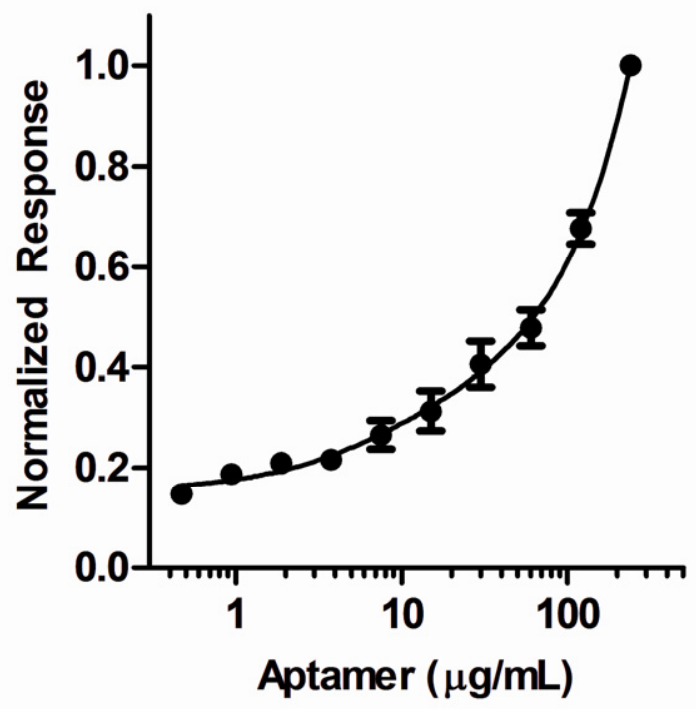




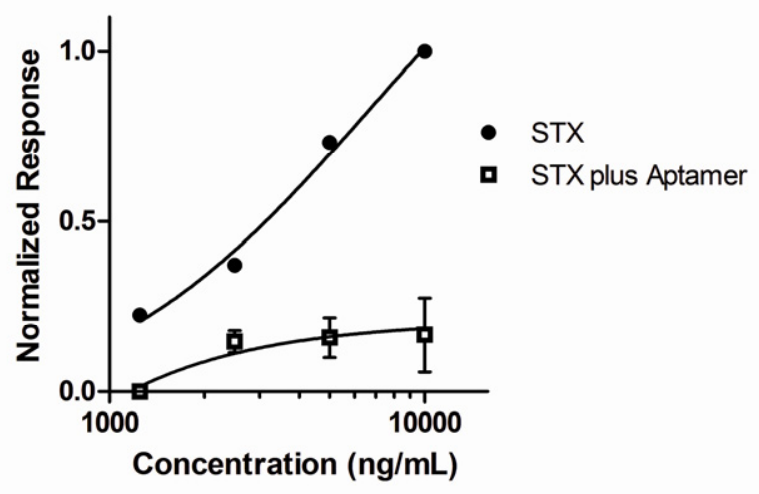

